\title{
Rare-earth quinolinates: \\ infrared-emitting molecular materials with a rich structural chemistry
}

Rik Van Deun*, Pascal Fias, Peter Nockemann, An Schepers, Tatjana N. Parac-Vogt, Kristof Van Hecke, Luc Van Meervelt and Koen Binnemans

\author{
Katholieke Universiteit Leuven, Department of Chemistry, \\ Celestijnenlaan 200F, B-3001 Leuven, BELGIUM. \\ * Address correspondence to this author. E-mail: \\ rik.vandeun@chem.kuleuven.ac.be
}

Supporting Information 
Table S1. CHN elemental analysis data of rare-earth quinolinates synthesised by different methods.

\begin{tabular}{|c|c|c|c|c|c|c|c|c|}
\hline \multirow[t]{2}{*}{ Compd } & \multirow[t]{2}{*}{$\begin{array}{l}\text { Synthetic } \\
\text { method }\end{array}$} & \multirow[t]{2}{*}{$\begin{array}{l}\text { Structure used to } \\
\text { calculate CHN data }\end{array}$} & \multicolumn{3}{|c|}{ Calculated \% } & \multicolumn{3}{|c|}{ Found \% } \\
\hline & & & $\mathrm{C}$ & $\mathrm{H}$ & $\mathrm{N}$ & $\mathrm{C}$ & $\mathrm{H}$ & $\mathrm{N}$ \\
\hline \multicolumn{9}{|c|}{ Reinvestigation of literature procedures } \\
\hline 1 & A & $\mathrm{Gd}\left(\mathrm{C}_{9} \mathrm{H}_{6} \mathrm{NO}\right)_{3} \cdot \mathrm{H}_{2} \mathrm{O}$ & 53.36 & 3.32 & 6.91 & 53.11 & 3.19 & 6.76 \\
\hline 2 & A altered & $\operatorname{Er}\left(\mathrm{C}_{9} \mathrm{H}_{6} \mathrm{NO}\right)_{3} \cdot 2 \mathrm{H}_{2} \mathrm{O}$ & 51.01 & 3.49 & 6.61 & 50.77 & 3.10 & 6.25 \\
\hline 3 & B & $\mathrm{Y}\left(\mathrm{C}_{9} \mathrm{H}_{6} \mathrm{NO}\right)_{3} \cdot 5 / 2 \mathrm{H}_{2} \mathrm{O}$ & 57.25 & 4.09 & 7.42 & 57.37 & 3.47 & 7.18 \\
\hline 4 & $\mathrm{C}$ & $\mathrm{Er}\left(\mathrm{C}_{9} \mathrm{H}_{5} \mathrm{ClNO}\right)_{3} \cdot \mathrm{H}_{2} \mathrm{O}$ & 44.97 & 2.38 & 5.83 & 44.77 & 1.98 & 5.36 \\
\hline 5 & $C$ & $\operatorname{Er}\left(\mathrm{C}_{9} \mathrm{H}_{4} \mathrm{Br}_{2} \mathrm{NO}\right)_{3}$ & 30.22 & 1.13 & 3.92 & & & \\
\hline 3 & $c$ & $\mathrm{NH}_{4}\left[\operatorname{Er}\left(\mathrm{C}_{9} \mathrm{H}_{4} \mathrm{Br}_{2} \mathrm{NO}\right)_{4}\right] \cdot \mathrm{H}_{2} \mathrm{O}$ & 30.64 & 1.57 & 4.96 & 30.86 & 1.34 & 4.48 \\
\hline 6 & $\mathrm{C}$ & $\mathrm{NH}_{4}\left[\mathrm{Y}\left(\mathrm{C}_{9} \mathrm{H}_{4} \mathrm{Cl}_{2} \mathrm{NO}\right)_{4}\right] \cdot \mathrm{H}_{2} \mathrm{O}$ & 44.25 & 2.27 & 7.17 & 44.38 & 2.20 & 6.50 \\
\hline 7 & A & $\begin{array}{c}\operatorname{Er}\left(\mathrm{C}_{9} \mathrm{H}_{4} \mathrm{Cl}_{2} \mathrm{NO}\right)_{3} \cdot \mathrm{H}_{2} \mathrm{O} \\
\mathrm{NH}_{4}\left[\operatorname{Er}\left(\mathrm{C}_{9} \mathrm{H}_{4} \mathrm{Cl}_{2} \mathrm{NO}\right)_{4}\right] \cdot 3 \mathrm{H}_{2} \mathrm{O}\end{array}$ & $\begin{array}{l}39.34 \\
39.61\end{array}$ & $\begin{array}{l}1.71 \\
2.40\end{array}$ & $\begin{array}{l}5.10 \\
6.42\end{array}$ & 39.09 & 2.00 & 4.63 \\
\hline
\end{tabular}

Improved methods

\begin{tabular}{|c|c|c|c|c|c|c|c|c|}
\hline 8 & Tetrakis & $\mathrm{Na}\left[\mathrm{Yb}\left(\mathrm{C}_{9} \mathrm{H}_{5} \mathrm{ClNO}\right)_{4}\right] \cdot \mathrm{H}_{2} \mathrm{O}$ & 46.57 & 2.39 & 6.03 & 46.62 & 2.26 & 5.69 \\
\hline 9 & Tetrakis & $\mathrm{Na}\left[\mathrm{Y}\left(\mathrm{C}_{9} \mathrm{H}_{4} \mathrm{Cl}_{2} \mathrm{NO}\right)_{4}\right] \cdot 1 / 2 \mathrm{H}_{2} \mathrm{O}$ & 44.44 & 1.76 & 5.76 & 44.28 & 1.48 & 5.45 \\
\hline 10 & Tris & $\mathrm{Y}\left(\mathrm{C}_{9} \mathrm{H}_{4} \mathrm{Cl}_{2} \mathrm{NO}\right)_{3} \cdot \mathrm{H}_{2} \mathrm{O}$ & 43.47 & 1.89 & 5.63 & 43.68 & 1.82 & 5.13 \\
\hline 3 & Trimer & {$\left[\mathrm{Y}_{3}\left(\mathrm{C}_{9} \mathrm{H}_{6} \mathrm{NO}\right)_{8}\right] \mathrm{Cl} \cdot 3 \mathrm{H}_{2} \mathrm{O}$} & 57.29 & 3.61 & 7.42 & 57.37 & 3.47 & 7.18 \\
\hline \multicolumn{9}{|c|}{ Samples used for spectroscopy } \\
\hline 11 & Tetrakis & $\mathrm{Na}\left[\operatorname{Er}\left(\mathrm{C}_{9} \mathrm{H}_{4} \mathrm{Cl}_{2} \mathrm{NO}\right)_{4}\right] \cdot 1 / 2 \mathrm{H}_{2} \mathrm{O}$ & 41.12 & 1.63 & 5.33 & 41.10 & 1.72 & 5.01 \\
\hline 12 & Tris & {$\left[\mathrm{Er}\left(\mathrm{C}_{9} \mathrm{H}_{4} \mathrm{Cl}_{2} \mathrm{NO}\right)_{3}\right]_{2} \cdot 2 \mathrm{H}_{2} \mathrm{O}$} & 39.34 & 1.71 & 5.09 & 39.24 & 1.70 & 4.69 \\
\hline 13 & Tetrakis & $\mathrm{Na}\left[\mathrm{Er}\left(\mathrm{C}_{9} \mathrm{H}_{5} \mathrm{ClNO}\right)_{4}\right] \cdot \mathrm{H}_{2} \mathrm{O}$ & 46.86 & 2.40 & 6.07 & 47.10 & 2.28 & 5.79 \\
\hline 14 & Tetrakis & $\mathrm{Na}\left[\operatorname{Er}\left(\mathrm{C}_{9} \mathrm{H}_{6} \mathrm{NO}\right)_{4}\right] \cdot \frac{1}{2} \mathrm{H}_{2} \mathrm{O}$ & 55.73 & 3.25 & 7.22 & 55.76 & 2.85 & 6.96 \\
\hline 15 & B & $\mathrm{Na}\left[\mathrm{Er}_{3}\left(\mathrm{C}_{9} \mathrm{H}_{6} \mathrm{NO}\right)_{8}\right] \mathrm{Cl}(\mathrm{OH}) \cdot 2 \mathrm{H}_{2} \mathrm{O}$ & 49.07 & 3.20 & 6.36 & 49.23 & 2.96 & 5.78 \\
\hline
\end{tabular}


Table s2. Crystallographic data for compounds 2, 4, 5 and $\mathbf{7}$.

\begin{tabular}{|c|c|c|c|c|}
\hline & Compound 2 & Compound 4 & Compound 5 & Compound 7 \\
\hline formula & C86H80ClEr3N9O18 & $\mathrm{C} 80 \mathrm{H} 49 \mathrm{Cl} 26 \mathrm{Er} 3 \mathrm{~N} 8 \mathrm{O} 10$ & C4 0H24Br8ErN5O6 & C39H36Cl6ErN3O11 \\
\hline formula wt & 2064.82 & 2705.75 & 1477.10 & 1102.67 \\
\hline cryst syst & triclinic & monoclinic & triclinic & monoclinic \\
\hline space group & P-1 (No.2) & $\mathrm{P} 21 / \mathrm{n} \quad(\mathrm{No} .14)$ & $\mathrm{P}-1 \quad$ (No.2) & P21 (No.4) \\
\hline $\mathrm{a}(\AA \AA \AA)$ & $12.3890(4)$ & $16.8271(6)$ & $11.8160(8)$ & $13.378(3)$ \\
\hline b $\quad(\AA)$ & $17.3230(5)$ & $28.8121(10)$ & $12.0722(7)$ & $24.139(5)$ \\
\hline C $(\AA)$ & $20.8452(5)$ & $20.1559(8)$ & $17.8093(10)$ & $13.622(3)$ \\
\hline$\alpha \quad(\operatorname{deg})$ & $111.719(2)$ & 90.000 & $85.354(3)$ & 90.000 \\
\hline$\beta \quad$ (deg) & $103.170(2)$ & $102.991(2)$ & $88.989(4)$ & $106.12(3)$ \\
\hline$\gamma($ deg $)$ & $97.164(2)$ & 90.000 & $61.002(4)$ & 90.000 \\
\hline $\mathrm{V}\left(\AA^{3}\right)$ & $3937.3(2)$ & $9522.0(6)$ & $2214.0(2)$ & $4226.0(17)$ \\
\hline $\mathrm{z}$ & 2 & 4 & 2 & 4 \\
\hline unique & 14354 & 18031 & 8182 & 8254 \\
\hline $\begin{array}{l}\text { reflections } \\
\text { observed } \\
\text { reflections }\end{array}$ & 10171 & 11511 & 5911 & 5718 \\
\hline$\left(F_{\circ}>4 \sigma\left(F_{\circ}\right)\right)$ & & & & \\
\hline $\mathrm{R}_{1}$ & 0.0490 & 0.0687 & 0.0551 & 0.0584 \\
\hline$\omega R_{2}^{a}$ & 0.1191 & 0.1684 & 0.1355 & 0.1306 \\
\hline $\mathrm{R}_{1}$ (all data) & 0.0728 & 0.1037 & 0.0740 & 0.1108 \\
\hline $\begin{array}{l}\omega R_{2}(a l l \\
\operatorname{data})\end{array}$ & 0.1303 & 0.1824 & 0.1464 & 0.1632 \\
\hline GooF & 0.937 & 0.954 & 0.934 & 1.007 \\
\hline
\end{tabular}




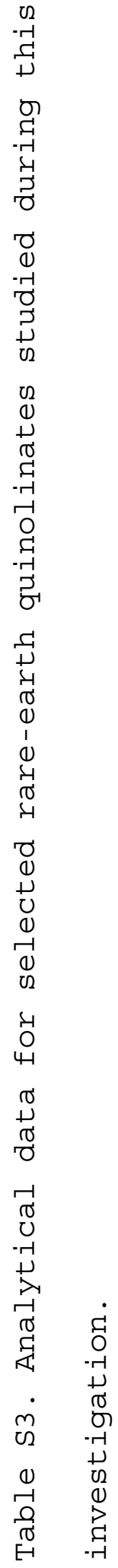

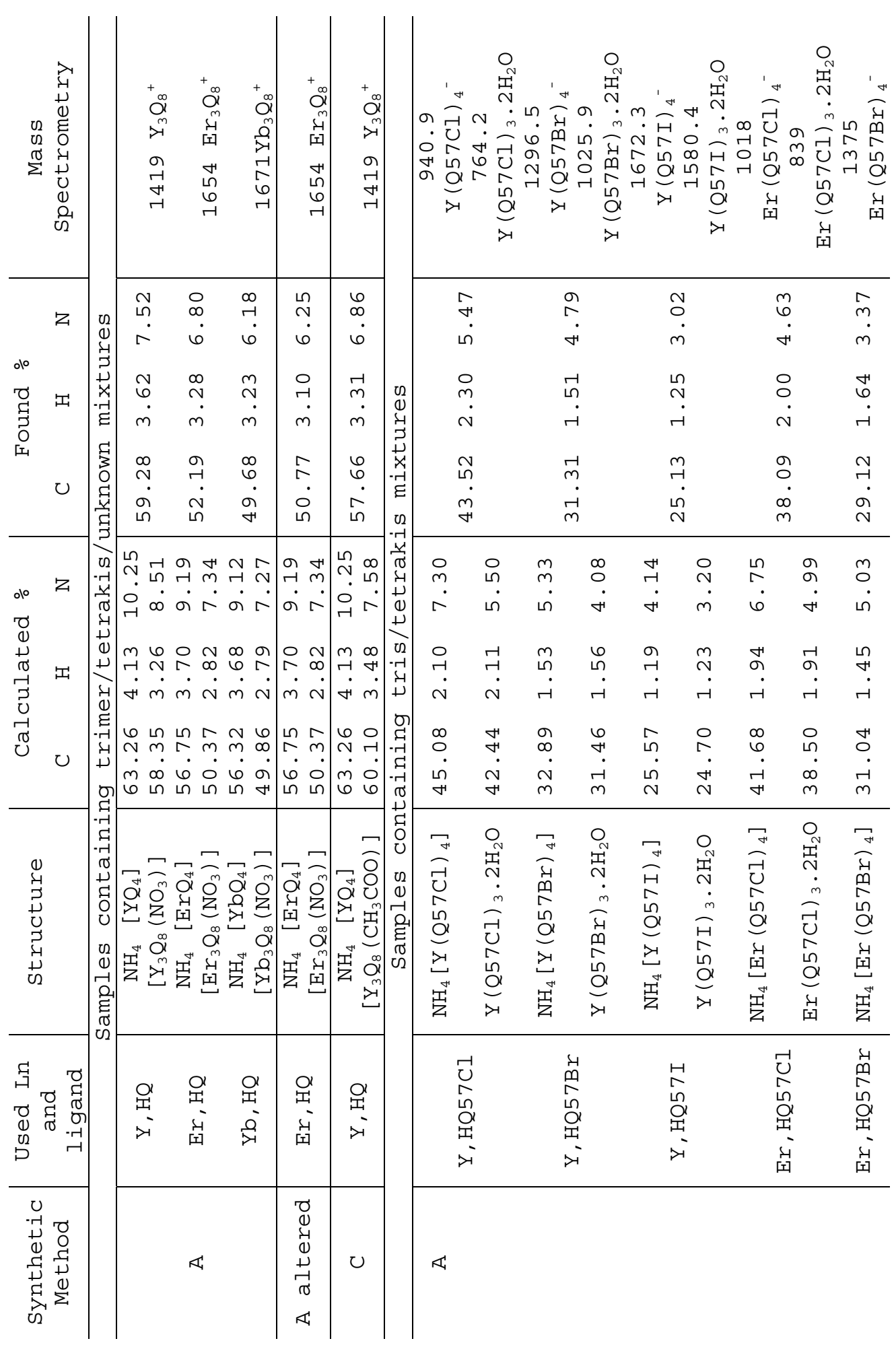




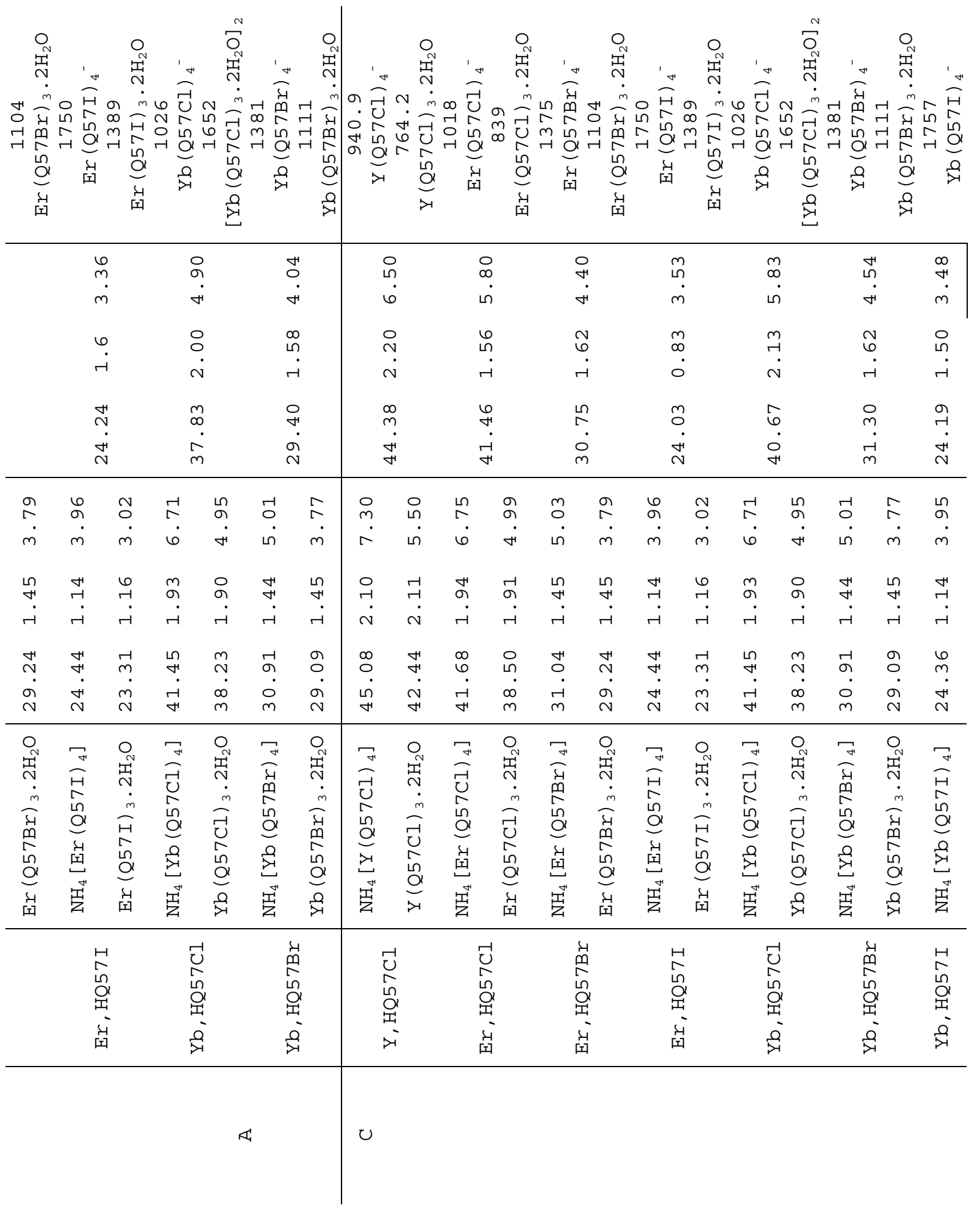




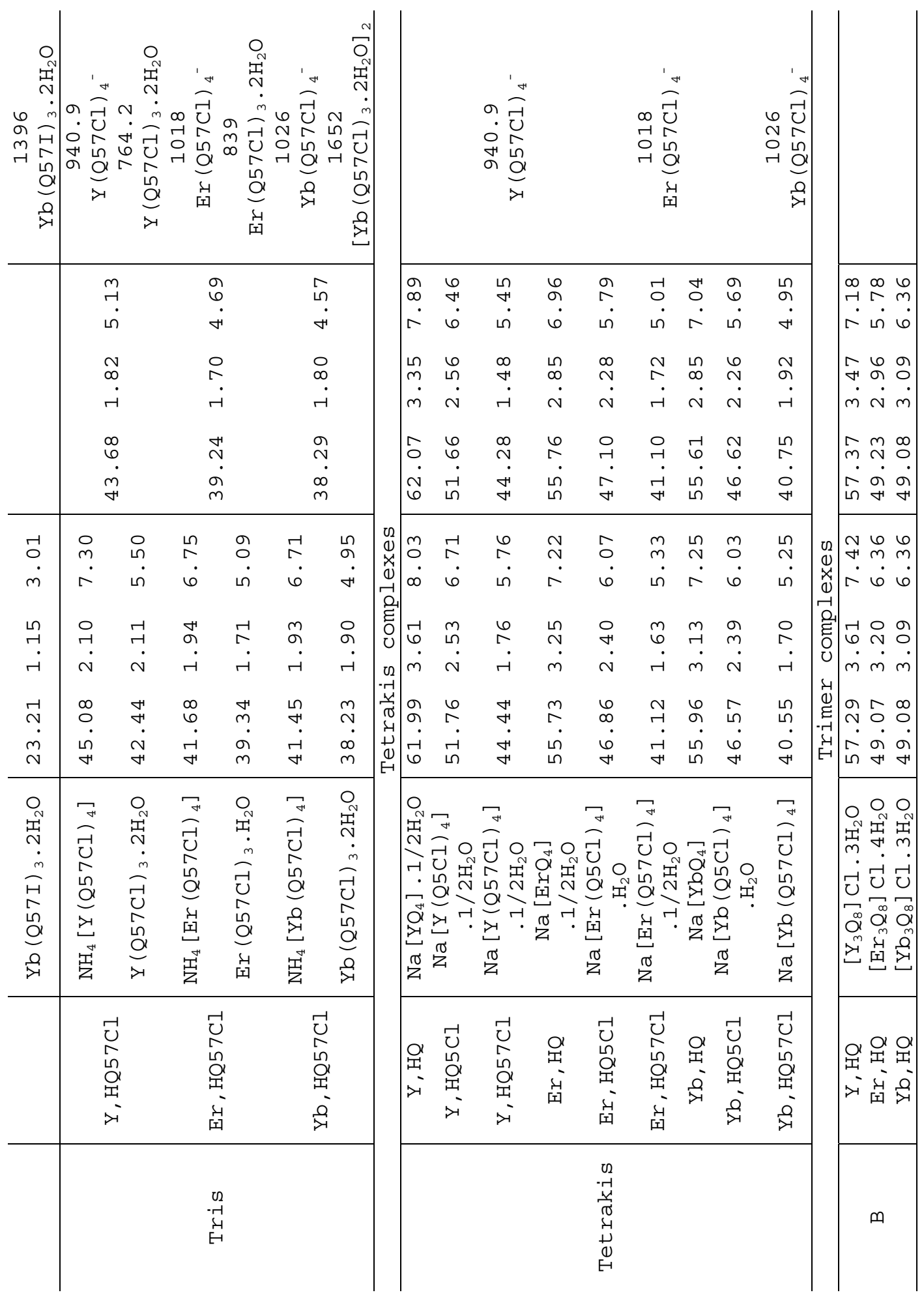


Figure S1. Crystal structure of $\mathrm{NH}_{4}\left[\mathrm{Er}_{3}(\mathrm{Q})_{8}\right] \mathrm{Cl}(\mathrm{OH}) \cdot 4 \mathrm{C}_{4} \mathrm{H}_{8} \mathrm{O}_{2}$ (compound 2) showing thermal ellipsoids. Solvent molecules (dioxane) have been omitted for clarity.

Figure S2. Crystal structure of $\mathrm{Er}_{3}(\mathrm{Q} 5 \mathrm{Cl})_{8}\left(\mathrm{CH}_{3} \mathrm{COO}\right) \cdot 6 \mathrm{CHCl}_{3}$ (compound 4) showing thermal ellipsoids. Solvent molecules (chloroform) have been omitted for clarity.

Figure S3. Crystal structure of $\mathrm{NH}_{4}\left[\mathrm{Er}(\mathrm{Q} 57 \mathrm{Br})_{4}\right] \cdot \mathrm{C}_{4} \mathrm{H}_{8} \mathrm{O}_{2}$ (compound 5) showing thermal ellipsoids. Solvent molecules (dioxane) have been omitted for clarity.

Figure S4. Crystal structure of $\operatorname{Er}(Q 57 \mathrm{Cl})_{3} \cdot 6 \mathrm{C}_{4} \mathrm{H}_{8} \mathrm{O}_{2}$ (compound 7) showing thermal ellipsoids. Solvent molecules (dioxane) have been omitted for clarity.

Figure S5. Spacefilling model of selected rare-earth complexes to indicate the 'cavity'. (a) Tetrakis complex 5 with halogen-atoms in the 7 position and an ammonium ion bound in the cavity. (b) Trimeric complex 2 of unsubstituted HQ. Because of the absence of any substituents in the 7-position, the cavity is large enough to incorporate a rare-earth ion, while this is not the case in (a). 
Figure S6. 2D-COSY ${ }^{1} \mathrm{H}-\mathrm{NMR}$ spectrum of compound 3 in DMSO-d 6 solution at room temperature. The protons in the ligand have been labeled from a to $\mathrm{f}$. There are four magnetically inequivalent pairs of ligand, which have been labeled 1 to 4 . Therefore, notation $f_{1}$ means proton $f$ of the ligand pair 1 , while for example notation $c_{3}$ means proton $\mathrm{c}$ of the ligand pair 3 . The complete assignment of the COSY spectrum was only possible after recording a $2 \mathrm{D}-\mathrm{NOESY}{ }^{1} \mathrm{H}-\mathrm{NMR}$ spectrum under the same conditions, in which cross-peaks between protons $\mathrm{c}$ and $\mathrm{d}$ of each ligand pair were observed.

Figure S7. ${ }^{1} \mathrm{H}-\mathrm{NMR}$ spectra of (a) pure tetrakis, (b) a trimer/tetrakis mixture and (c) the pure trimeric form of the yttrium(III) complex of HQ. Spectrum (b) contains three species: trimer, tetrakis and a third unknown compound. This (b) is a typical NMR spectrum of a reaction product of literature synthesis of rare-earth complexes with unsubstituted HQ. The pure trimer (c) was synthesised according to method $B$.

Figure s8. Typical mass spectrum of a trimeric species. In this case, an yttrium(III) complex synthesised from HQ was analysed. The peak at $\mathrm{m} / \mathrm{z} 1419$ originates from the $\mathrm{Y}_{3} \mathrm{Q}_{8}{ }^{+}$fragment. 
Figure S9. Typical mass spectrum of a tris/tetrakis mixture. In this case, an erbium(III) complex synthesised from HQ57Cl was analysed. The tris fragments are located at $\mathrm{m} / \mathrm{z} 839$ (monomer $\operatorname{Er}(\mathrm{Q} 57 \mathrm{Cl})_{3} \cdot 2 \mathrm{H}_{2} \mathrm{O}$ ) and $\mathrm{m} / \mathrm{z} 1643$ (dimer $\operatorname{Er}_{2}(\mathrm{Q} 57 \mathrm{Cl})_{6} \cdot 2 \mathrm{H}_{2} \mathrm{O}$ ) while the tetrakis fragments are located at $\mathrm{m} / \mathrm{z} 1020$ (Er $(\mathrm{Q} 57 \mathrm{Cl})_{4}{ }^{-}$)

Figure S10. Fitted luminescence decay curve of compound 11 $\mathrm{Na}\left[\operatorname{Er}(Q 57 \mathrm{Cl})_{4}\right] \cdot 1 / 2 \mathrm{H}_{2} \mathrm{O}$ in DMSO $\left(\mathrm{C} \approx 10^{-5} \mathrm{~mol} \mathrm{~L} \mathrm{~L}^{-1}\right)$, excited at $355 \mathrm{~nm}$ (3rd Nd:YAG harmonic) at room temperature. Lifetime $\tau=2.3 \mu \mathrm{s}$. Figure S11. Excitation spectrum of $\mathrm{Na}\left[\mathrm{Yb}(\mathrm{Q} 57 \mathrm{Cl})_{4}\right]$ in $\mathrm{DMSO}(\mathrm{C} \approx$ $\left.10^{-5} \mathrm{~mol} \mathrm{~L}^{-1}\right)$, monitored at the emission maximum. The excitation spectrum closely resembles the absorption spectrum. The excitation spectra of the Er-analogues look very similar, but because of the lower luminescence intensities, the signals show more noise. 
Figure S1.

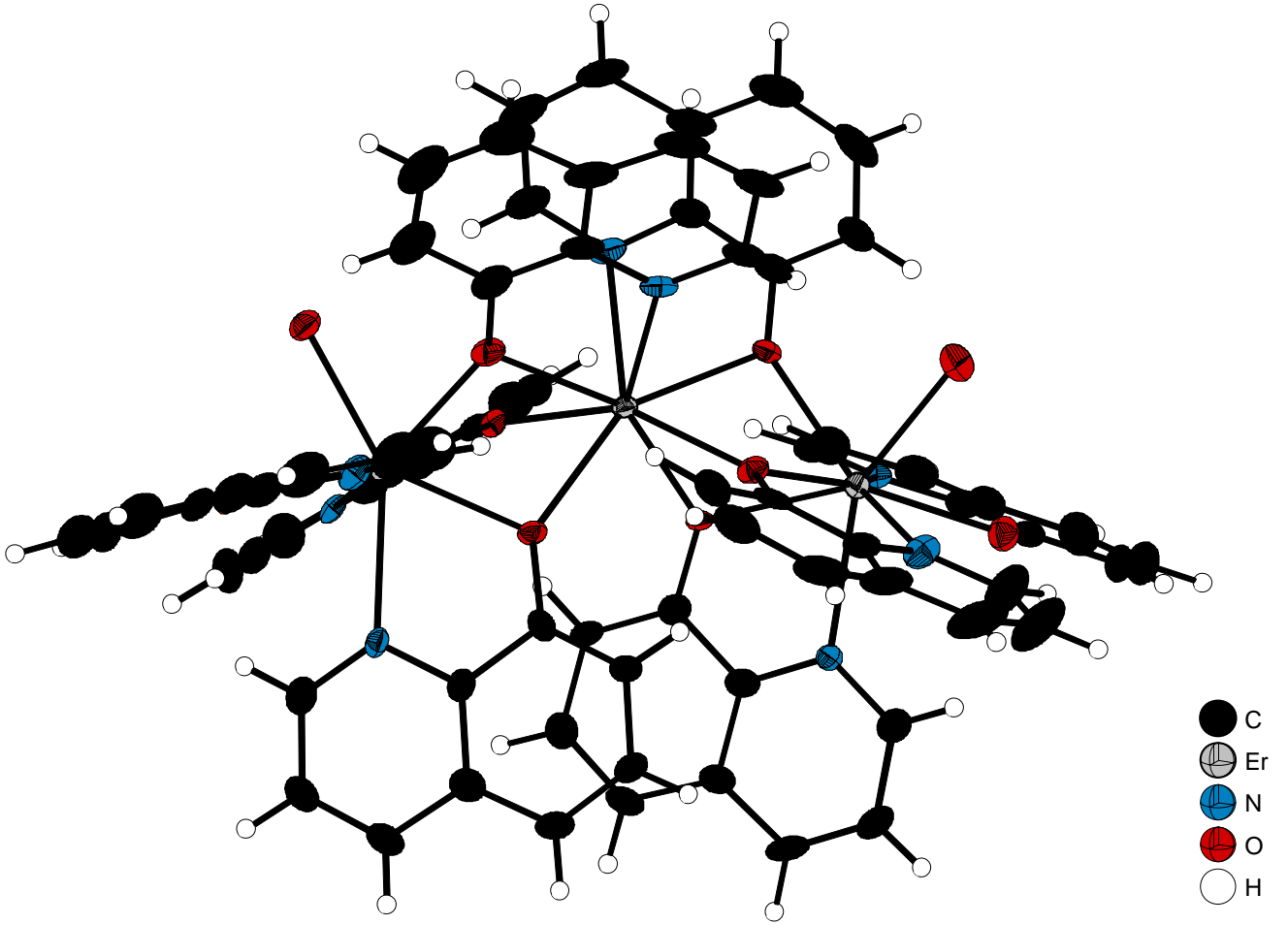


Figure S2.

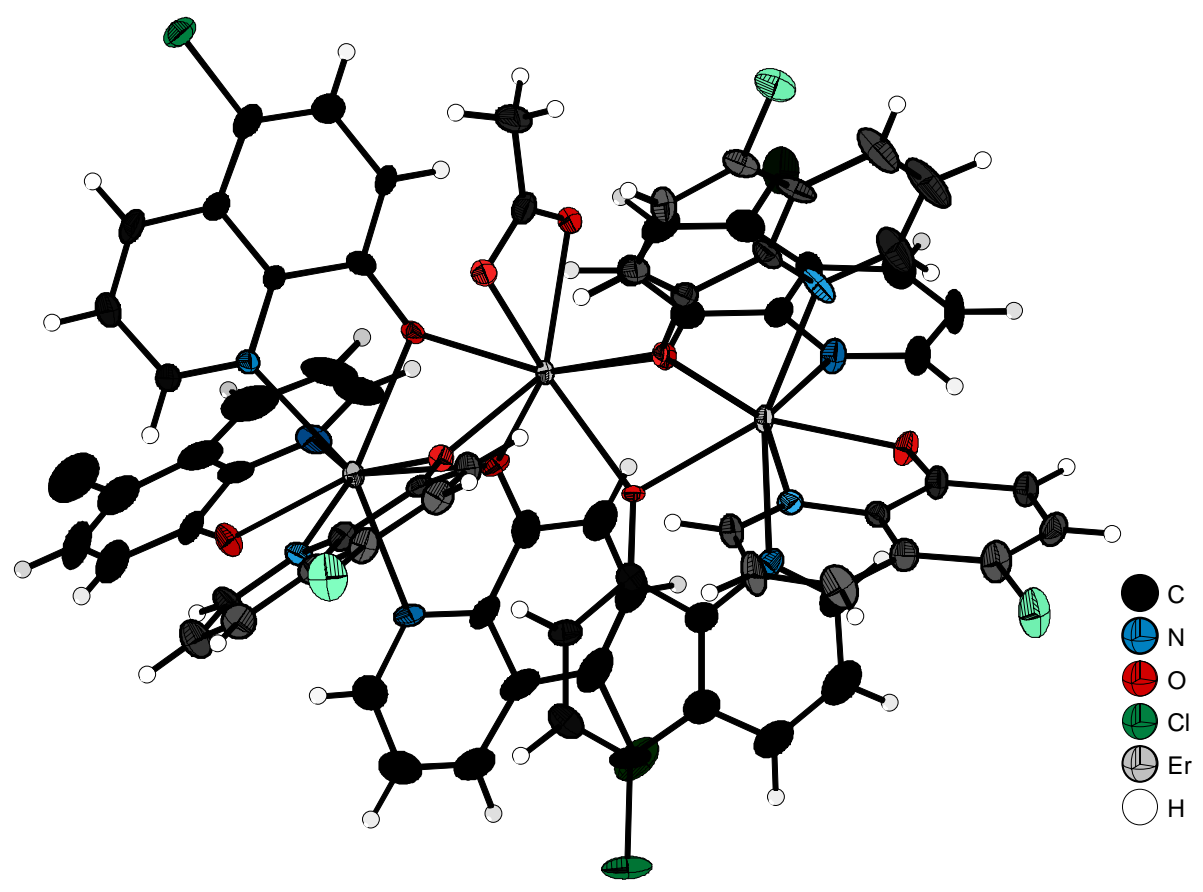


Figure S3.

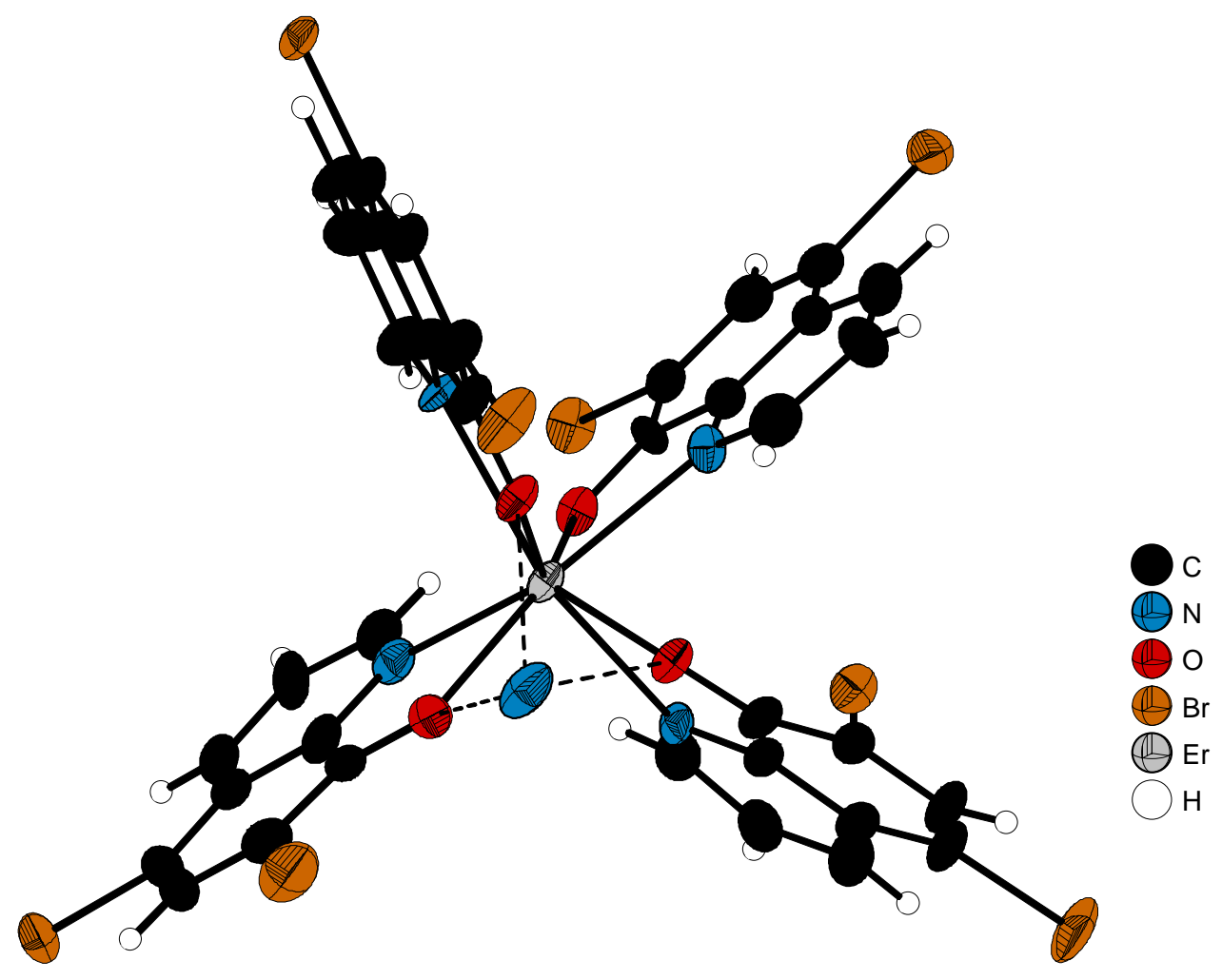


Figure S4.

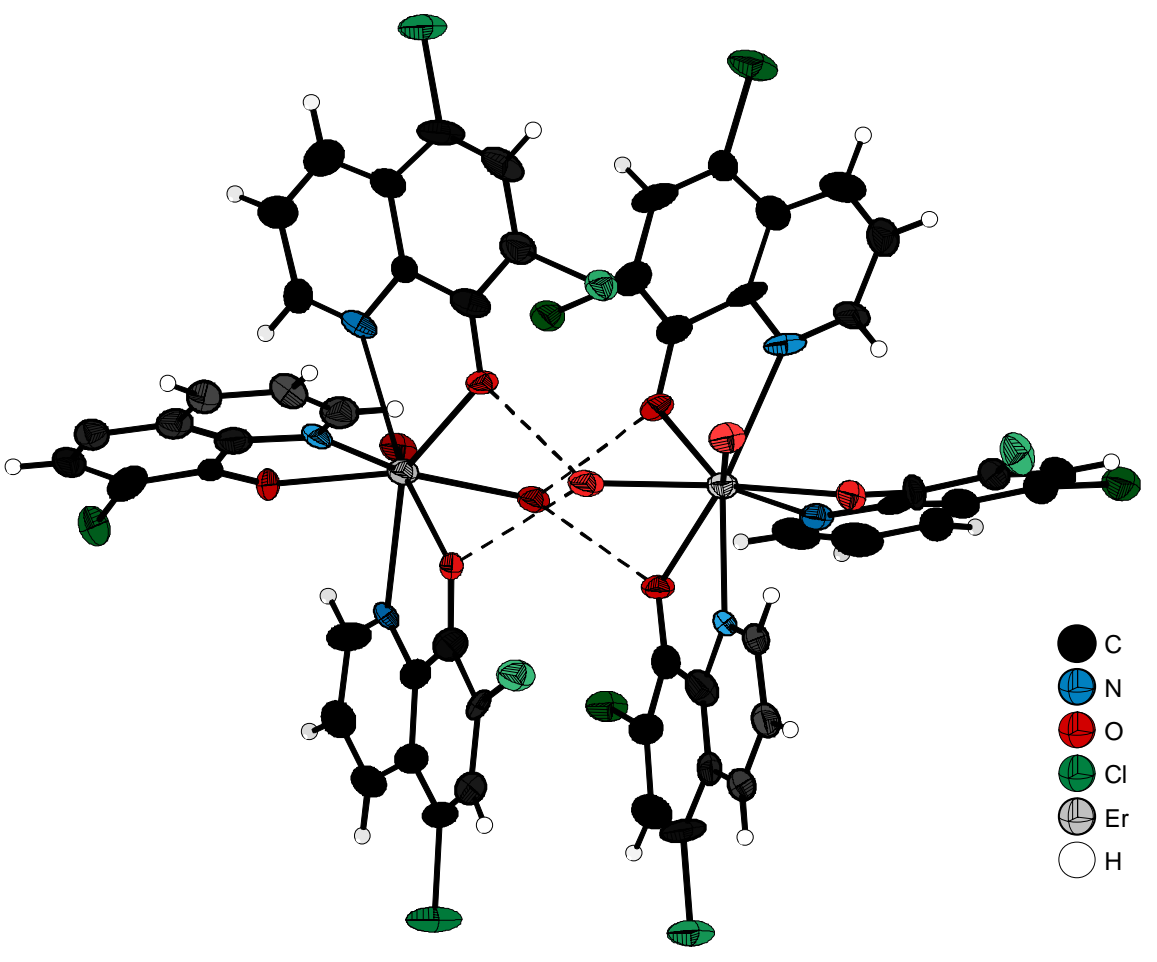


Figure S5.

(a)

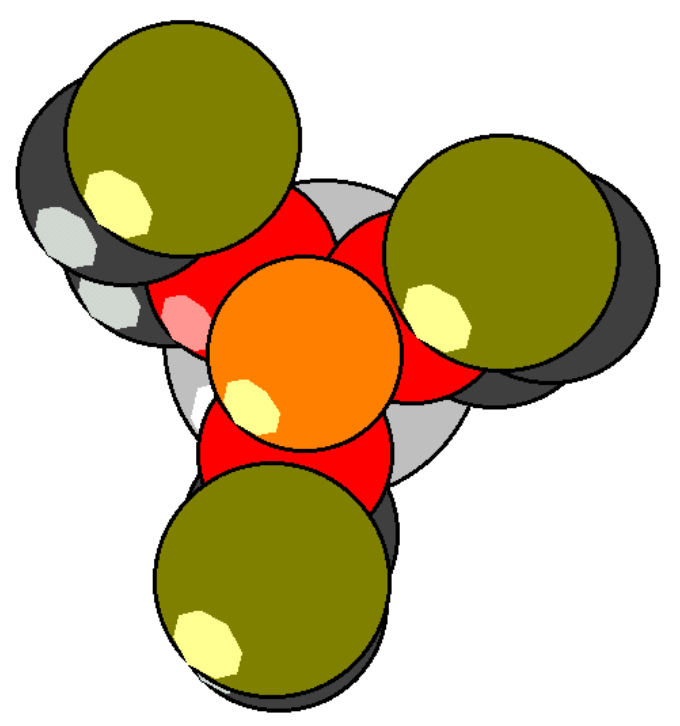

(b)

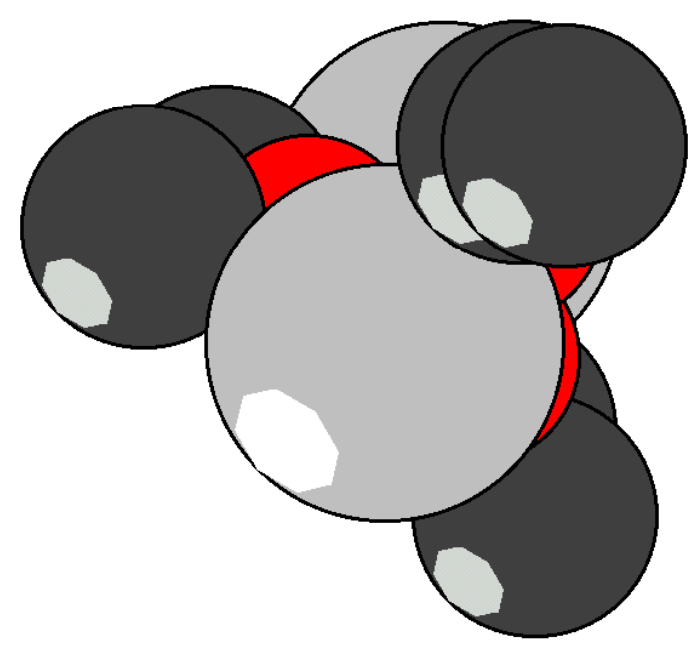


Figure S6.
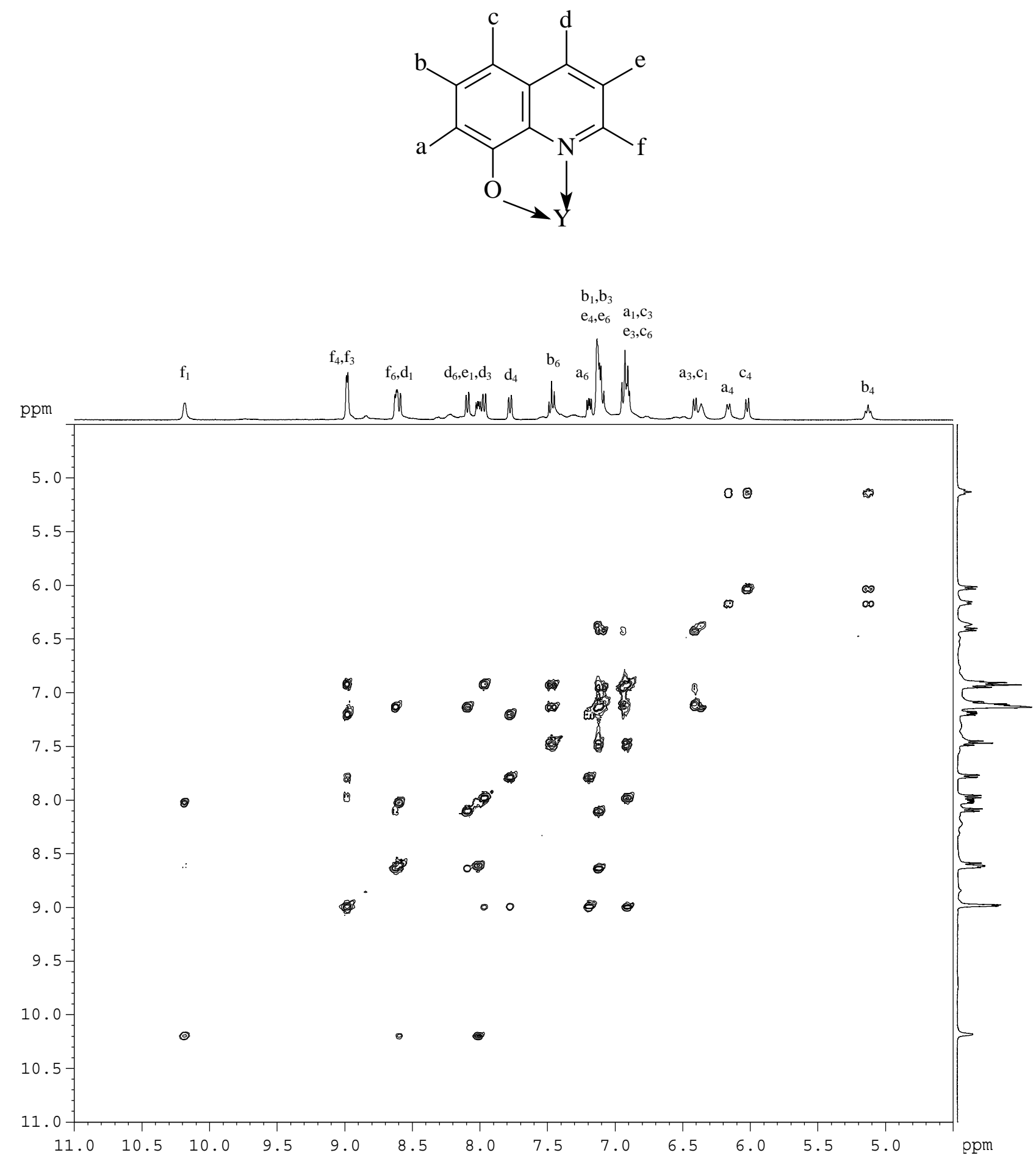
Figure S7.
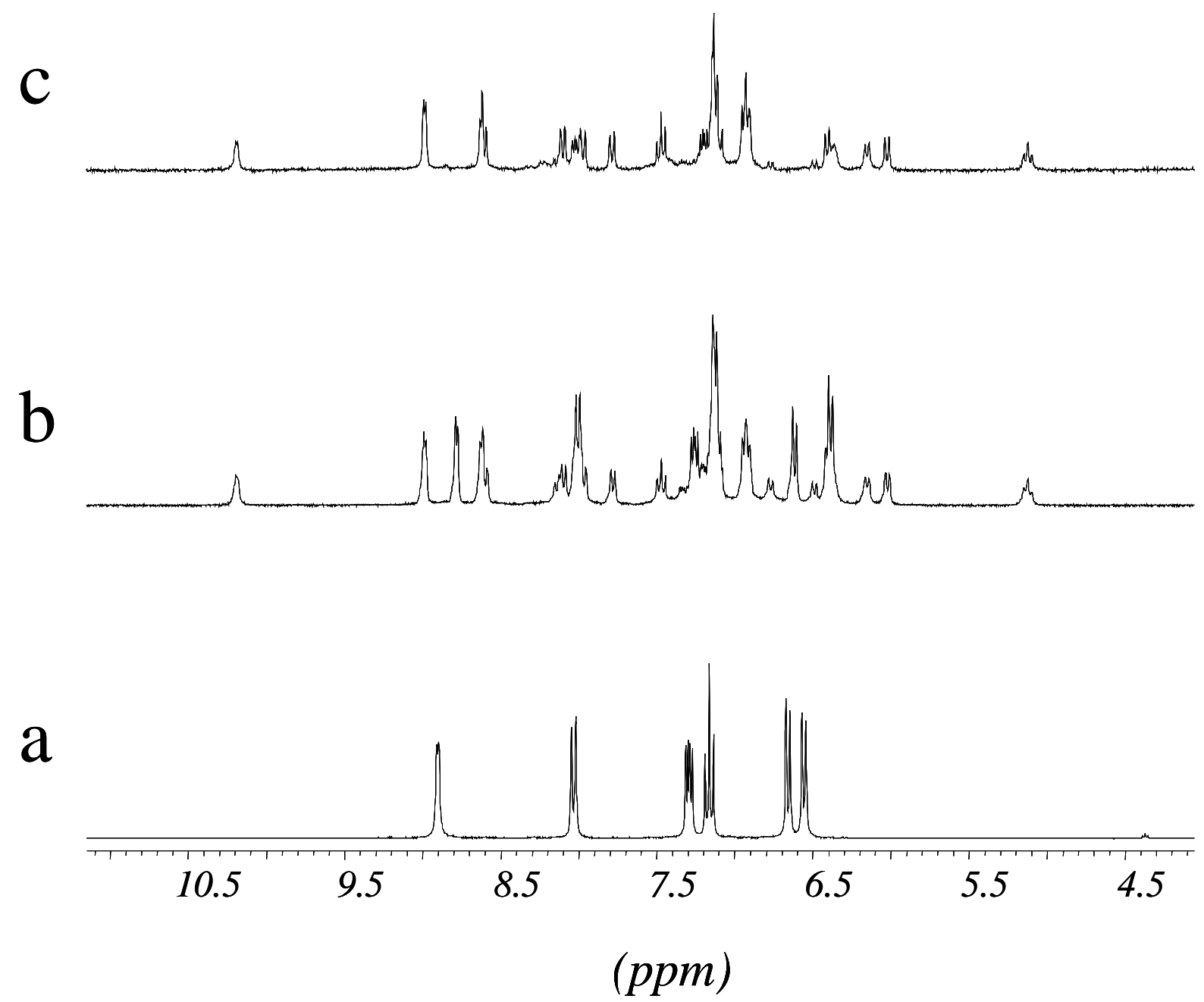


\section{Figure S8.}

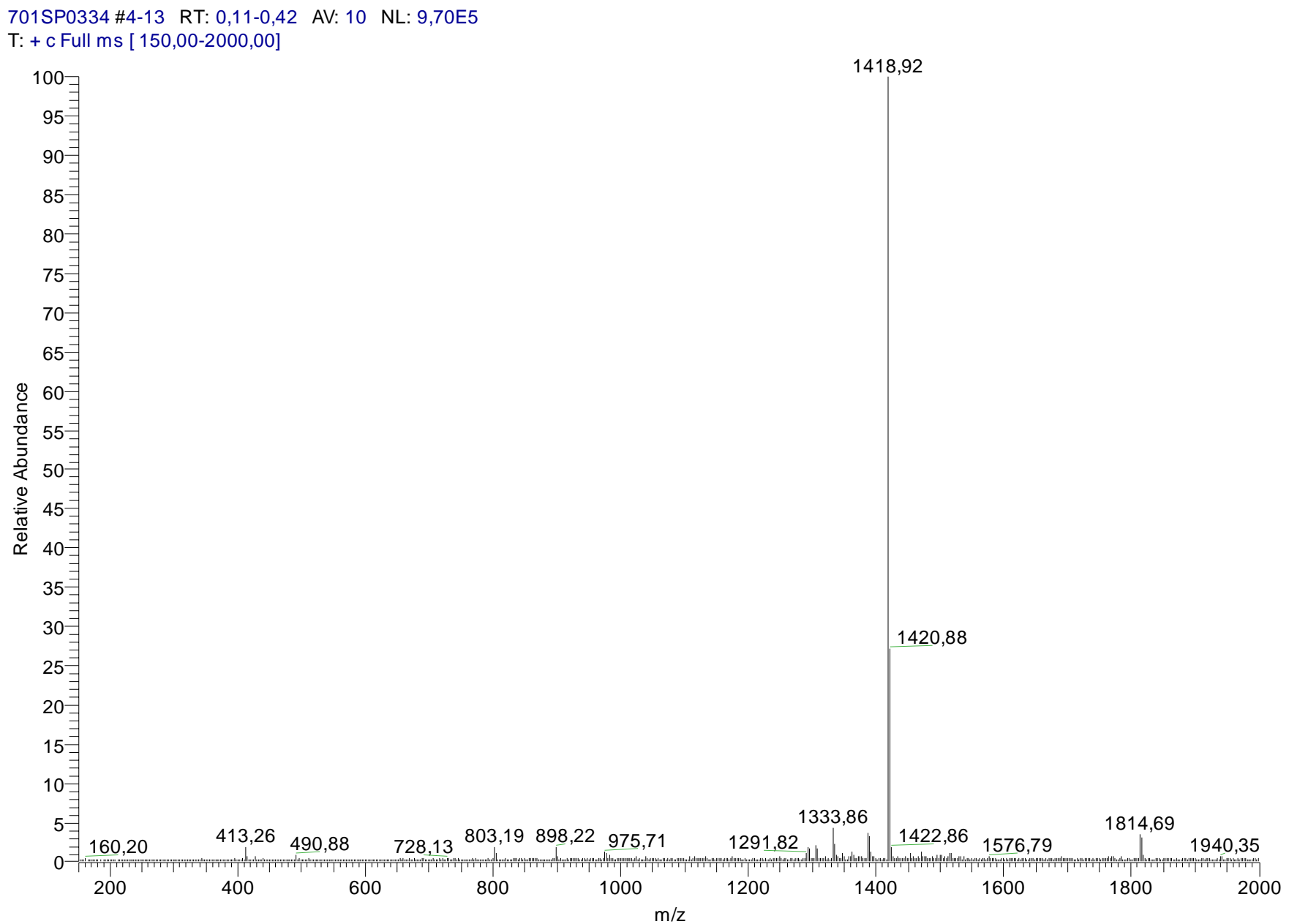




\section{Figure S9.}

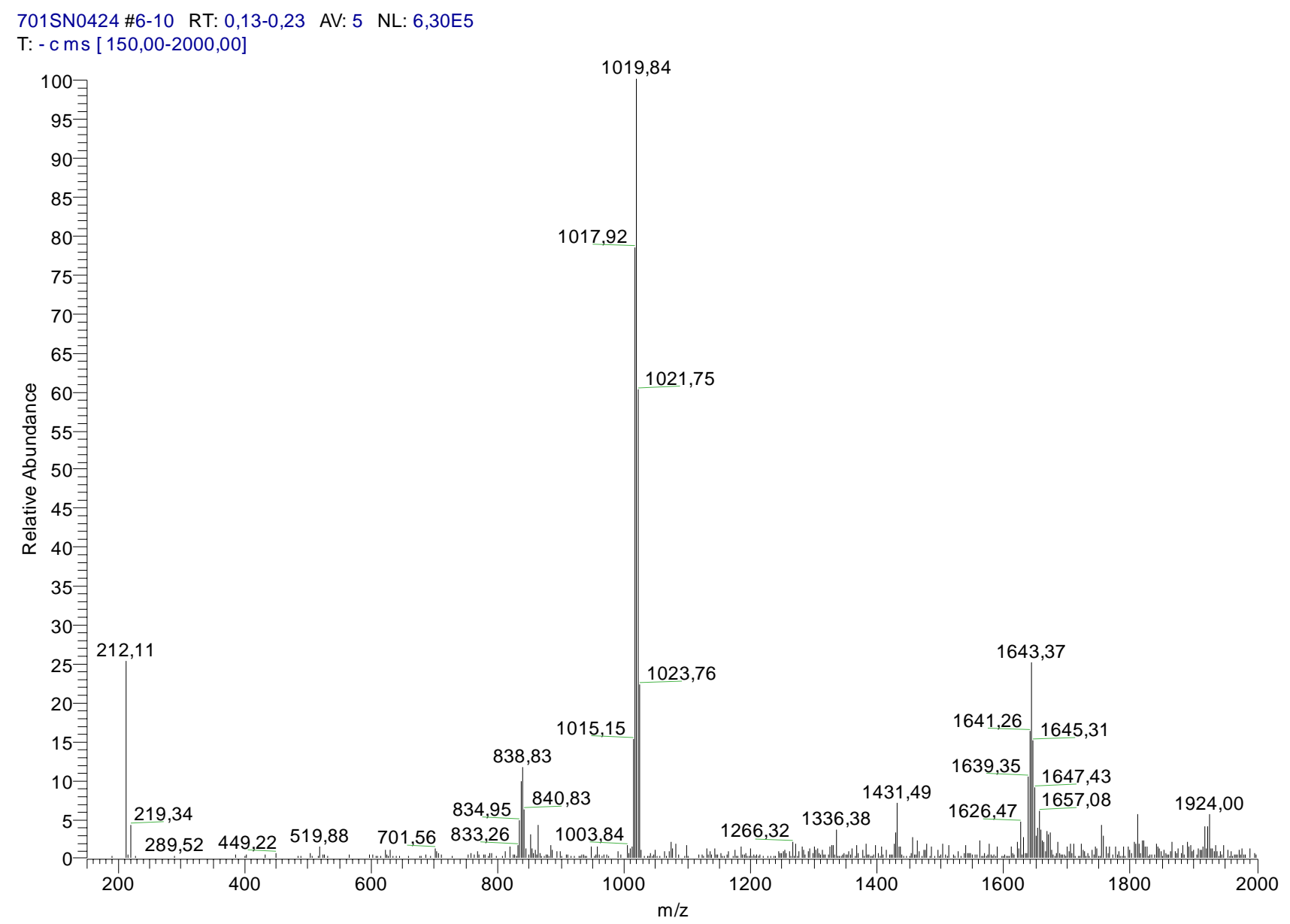


Figure S10.

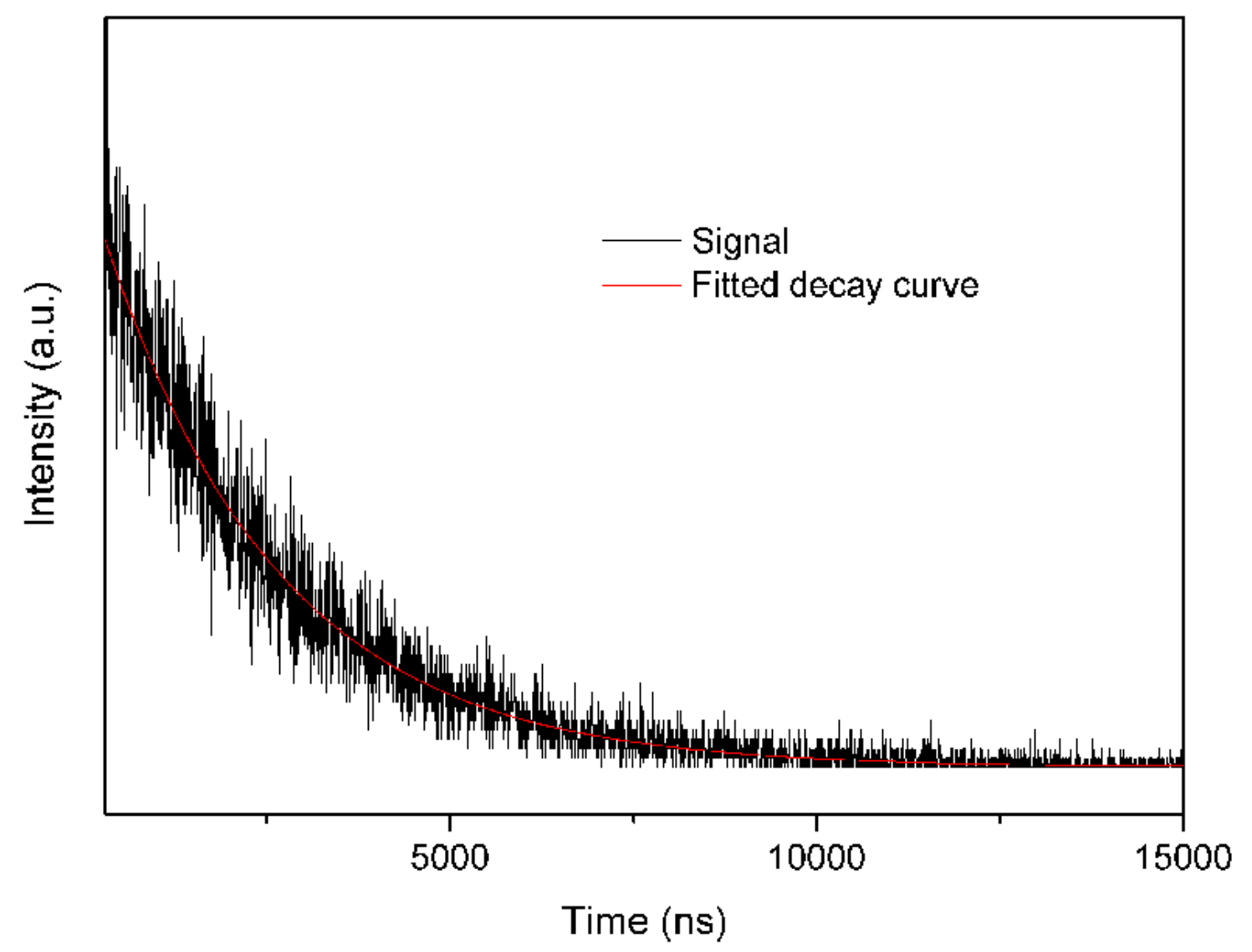


Figure S11.

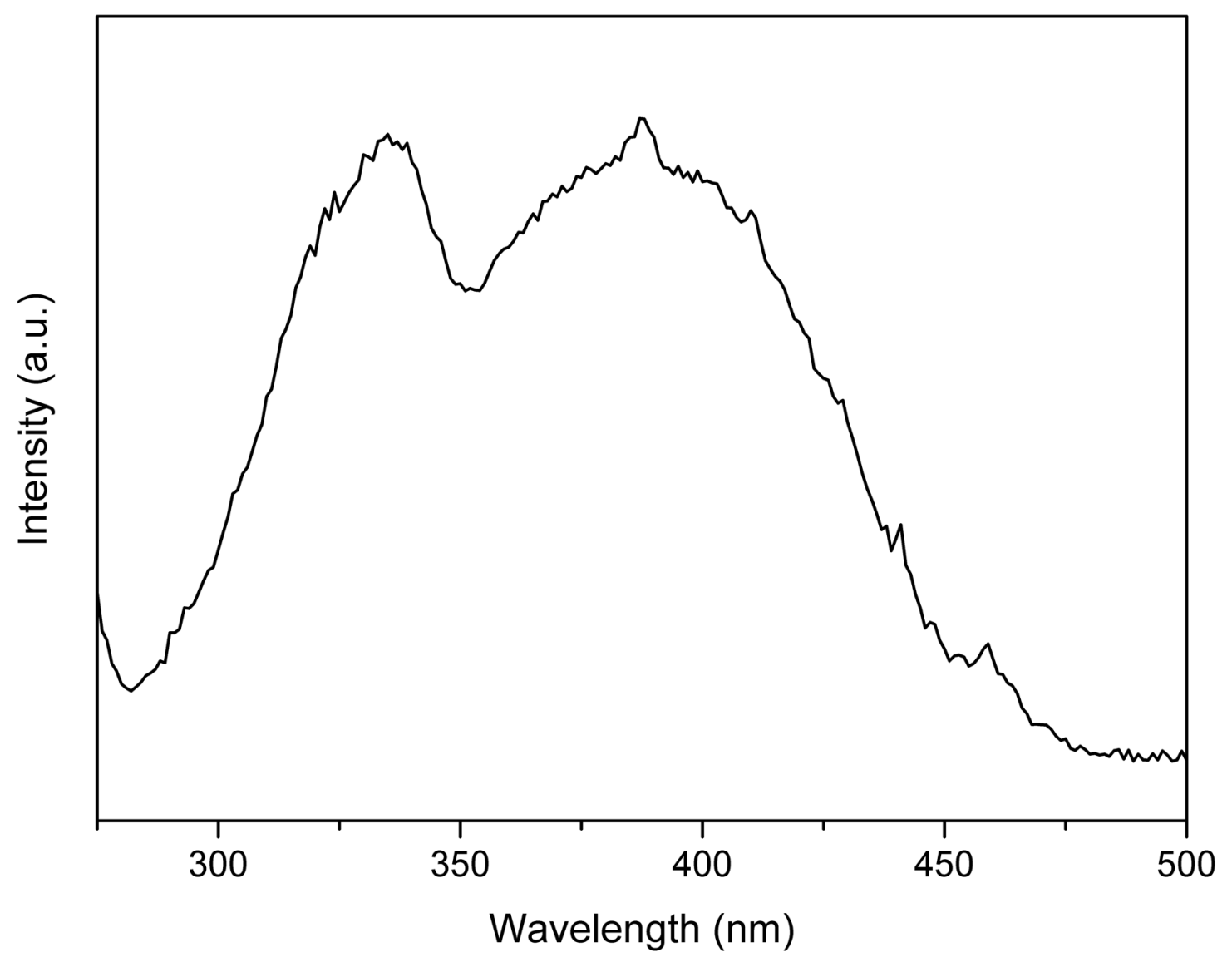

